\title{
Analysis on Energy Saving and Consumption Reducing of Wireless Sensor Network
}

\author{
Xiaoqing Yang \\ Mechanical and Electrical Engineering Department \\ Chongqing Vocational College of Industry and Commerce \\ Chongqing, China \\ e-mail: yangxiaoqing2014@yeah.net
}

\begin{abstract}
Through careful research and analysis on wireless sensor network (WSN), this paper confirms the important function of MAC protocol on wireless network communication. Moreover, through OPNET simulation software, this paper simulates different working scenes, and carefully researches MAC layer in IEEE 802.15.4 protocol. The data comparison result shows that GTS mechanism has the lowest energy consumption and it can delay the network lifetime in actual work.
\end{abstract}

Keywords-wireless sensor network (WSN); low power consumption; MAC protocol; energy saving.

\section{INTRODUCTION}

At current stage, the research and application of wireless sensor network (WSN) are on the basis of traditional wireless network and mobile ad hoc networks; actually, there exists difference. Due to the particularity of WSN, various kinds of problems will be met while those traditional methods are applied in the research process, and the most prominent problem is energy-saving problem. The current technology sensor node energy can only guarantee the use of small quantity of nodes; when the monitoring environment is poor or great quantity of nodes are arranged, the energy consumption will increase in a proportional way and the node energy will be quickly consumed. Under the condition of no supply of new energy, the monitoring network will immediately interrupt. Therefore, how to improve the lifetime of monitoring under condition of current sensor network energy technology is the biggest difficulty which shall be solved in the development of WSN.

While we carry out design of WSN according to requirement of energy saving and consumption reducing, we shall take the form and amount of energy consumption of different nodes as restraint and adopt integrated energy saving mode to carry out respective optimization from perspective of data processing and data transmission. The common design methods include the combination of software and hardware, and cross-layer network protocol, etc. At current stage, the energy saving research mainly focuses on data link layer and network layer in data transmission, and the data processing is rarely involved. The common understanding of data link is the channel for data transmission. The physical layer can realize data communication through long-term media and connection of limited lifetime. In the effective lifetime, the data communication can be freely carried out among terminal equipments, and the data receiving and dispatching relation formed via this method is called data link. At current stage, the research of energy saving technology of WSN mainly focuses on MAC protocol, which is the key point which guarantees the performance and efficient communication of sensor network.

The research focus at current stage is to design efficient router protocol to extend the lifetime of network system. Due to many particularities of WSN, such as high node density, complicated dynamic topological structure, and resource limitation, it is particular compared to other wireless networks, thus it is unable to directly transplant and apply the router protocol in other wireless network. In recent years, a foreign company has developed a protocol special for WSN; although this solves the difficulty of wireless network transmission to some degree, the protocol is one-sided and can't fundamentally solve the difficulty, thus it has weak practicability and can't be widely applied.

\section{ANALYSIS ON NODE ENERGY CONSUMPTION OF WSN}

The node of WSN is mainly composed of 4 parts: power module, control module, sensor module, and communication module; in the working process, the energy consumption mainly originates from 3 operations: wireless transmission, sensing, and processing.

\section{A. Control module}

The main function of control module is to carry out effective processing for various kinds of data information collected in sensor node according to design requirement; various kinds of command control processing of microprocessor need to consume large quantity of energy, and there is great difference in the energy consumption of micro-processor under operation state and dormant state; the energy consumption under dormant state is much lower than that under operation state. The energy consumption of control module under operation state is much lower than that under communication state. For example, while 100MIPS/W micro-processor executes $3,000,000$ commands at the same time, the energy 
consumption is basically equal to the energy consumption under the condition of transmitting $1 \mathrm{~KB}$ data at $100 \mathrm{~m}$ distance. In case of numerous network nodes, there will be numerous data information which shall be collected; at this time, through adding the working energy consumption of small quantity of control modules, it is able to meet the energy demand of large quantity of network data communication. Therefore, this is an important research direction for reducing the energy consumption of WSN at current stage.

\section{B. Sensor module}

The converter, A/D conversion, and front-end processing are the main position of energy consumption of sensor module, and the amount of energy consumption depends on the type of sensor. The sensor can be divided into 3 types [2]: (1) low energy-consumption sensor, including acceleration sensor and humidity sensor, etc.; (2) middle energy-consumption sensor, including magnetic sensor, etc.; (3) high energy-consumption sensor, including image sensor, and video sensor, etc. Besides, the energy consumption of sensor is in direct proportion to sensitive time, that is to say, the longer the sensitive time is, the larger the energy consumption will be. When the sensing environment is under serious disturbance, due to low quality of data collected, it is required to carry out repeated monitoring, thus the energy consumption will dramatically increase. However, under any condition, the energy consumption of sensor module is much lower than that of communication module.

\section{Communication module}

Due to rapid development of electronic information technology, the energy consumption can be greatly reduced through application of integrated circuit at current stage. The sensor module and control module in WSN adopt integrated circuit technique, thus their energy consumption can be almost ignored compared to the energy consumption of wireless communication module. Under normal operation, there is difference in the energy consumption of communication module based on operation state, that is to say, the specific energy consumption will be determined according to the operation state of module (receiving, sending, idle, dormant).

\section{ANALYSIS ON MAC PROTOCOL UNDER OPNET SIMULATION}

MAC layer protocol shall be designed according to the actual service environment of WSN, and there are 3 indexes used to evaluate the performance of MAC layer protocol: network efficiency, expandability, and node energy consumption. The author applies OPNET simulation to establish a small star-type network, makes use of simulation to set up different working scenes, and carefully researches the guaranteed time slot (GTS) and superframe structure in MAC protocol of IEEE 802.15.4 of LR-WPAN. Through analysis on energy consumption data acquired under simulation condition, it proves that GTS can greatly reduce network energy consumption.

\section{A. Analysis on MAC layer protocol}

1) MAC layer

MAC layer protocol mainly includes 2 parts: MAC layer data service and MAC layer management service. MAC layer data service ensures that MAC protocol data units can be accurately received and transmitted in physical layer data service, and MAC layer management service ensures that the database which saves the information of MAC layer protocol is under stable operation.

\section{2) Superframe communication}

Under the condition of IEEE 802.15.4, we take the LRWPAN network equipment communication whose periodic structure is superframe as research object. The beacon transmitted from network coordinator is set as the beginning of each superframe, and the information such as working time of superframe and the distribution within period of time shall be included in the beacon. After the receiving equipment in the network receives the beacon of initial superframe, it starts to enter into operation state according to the signal content in the beacon and continues this state until the superframe signal ends.

The communication time in superframe is divided into 2 parts: active time and inactive time. The superframe duration and beacon frame spacing signal are determined by superframe order (SO) and beacon order (BO), and the expression formula is shown as below:

$$
B I=\text { aBaseSuperframeDuration } \cdot 2^{B O} \quad(0 \leq B O \leq 14)
$$

$S D=$ aBaseSuperframeDuration $\cdot 2^{S O}(0 \leq S O \leq B O \leq 14)$

In the formula, aBaseSuperframe stands for minimum operation time of superframe, which is equivalent to $\mathrm{SO}=$ 0 .

While the data of physical layer is processed, MAC layer requires a limited time. When it is required to confirm the 1st transmission, the time interval between the 2nd transmission and ACK (acknowledgement frame) shall be not less than IFS. The capacity of transfer frame determines the size of IFS. Under the condition of aMaxSIFSFrameSize=144bits and aMaxPHYPacketSize=1016 bits, when the length of frame is less than 144 bits, IFS interval is 48 bits; when the length of frame is more than 144 bits and less than 1,016 bits, IFS interval is 160 bits.

\section{B. Establishment of OPNET simulation}

OPNET Modeler is a simulation software which is widely applied in WSN analysis; it can carry out real-time simulation on many kinds of actual service environments and carry out real-time and accurate data analysis. The author adopts OPNET simulation to construct small startype network which meets following conditions: scene scale: $10 \mathrm{~m} * 10 \mathrm{~m}$; quantity of wireless sensor nodes: 8 ; mode of communication: single-jump mode for PAN coordinator and node. 
IEEE 802.15.4 model is composed of following functional blocks: (1)MAC layer adopts GTS mechanism, and the data content of application layer is sent to the network after it is saved; (2) the physical layer mainly includes wireless receiver and wireless transmitter; (3)the data content of application layer includes following 3 types: Traffic Source, GTS Traffic Source, and Traffic sink; (4)as for power part, the energy consumption and the data of remaining energy are calculated.

\section{SIMULATION EXPERIMENT RESEARCH}

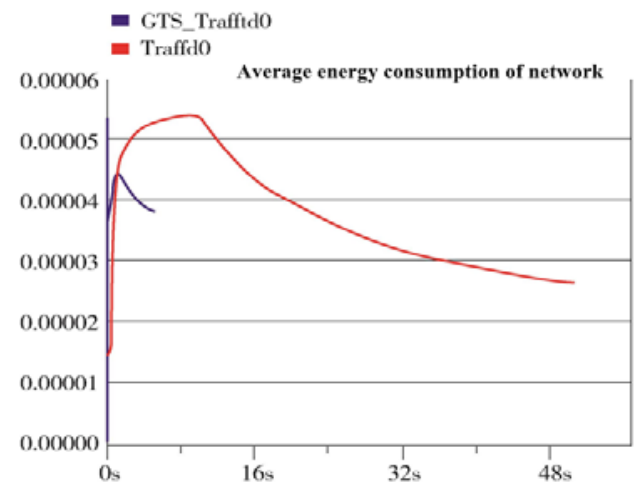

Figure 1. Comparison on average energy consumption of network

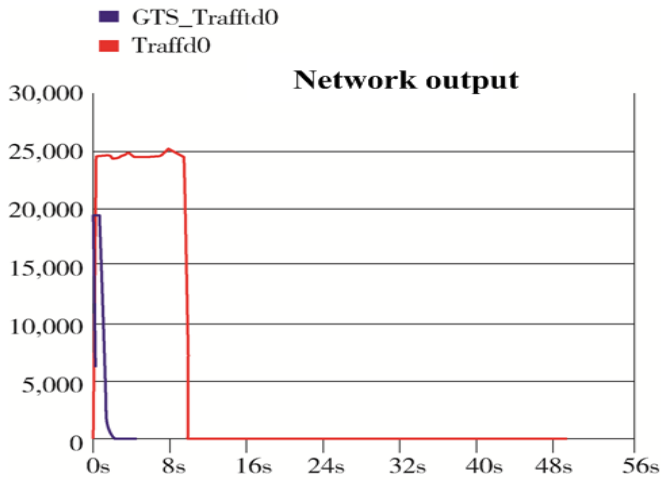

Figure 2. Comparison on network output

Figure 2 reflects the network output under 2 scenes, that is, the network output of GTS Traffic0 scene which adopts GTS mechanism is lower than the network output of Traffic0 scene. From Figure 1, it can be seen that the average energy consumption of network in Traffic 0 is lower than that in GTS_Traffic0 under the condition of $\mathrm{SO}=\mathrm{BO}=0$. Therefore, while the network is set, it is able to determine the communication mechanism after reasonably designing the network output and average energy consumption of network according to actual situation. From Figure 1 and 2, it can be seen that the average energy consumption of network in Figure 1 and the network output in Figure 2 are improved accordingly while $\mathrm{SO}=\mathrm{BO}$ is improved, and the proportion of Traffic data is improved; as for GTS_Traffic data, while $\mathrm{SO}=\mathrm{BO}$ is improved to intermediate stage, the average energy consumption of network approaches to end.

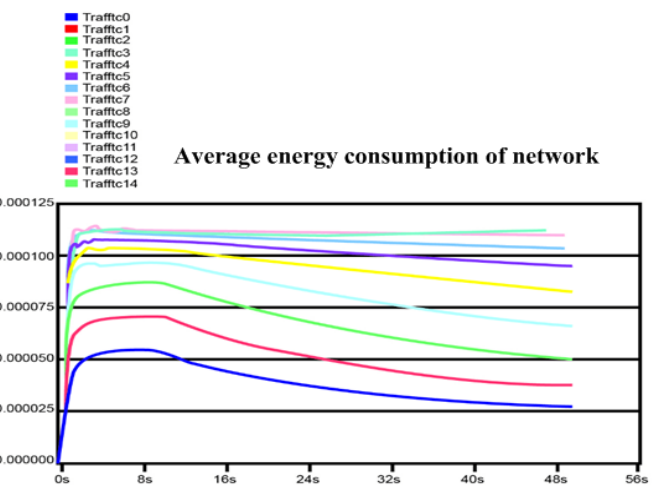

Figure 3. Average energy consumption of network under Traffic scene

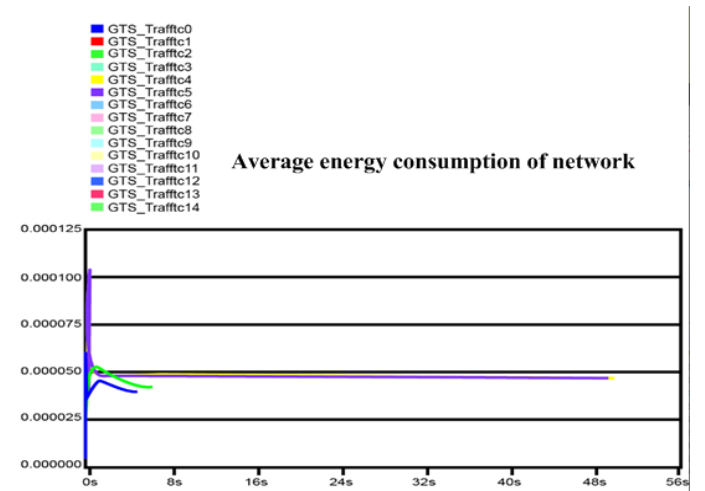

Figure 4. Average energy consumption of network under GTS Traffic scene

Figure 3 and 4 are the comparison diagram for average energy consumption of network under Traffic scene and GTS Traffic scene under different conditions $(\mathrm{SO}=\mathrm{BO}=0 \sim \mathrm{SO}=\mathrm{BO}=14)$. With the increase of $\mathrm{SO}=\mathrm{BO}$, the average energy consumption of network under above 2 scenes shows an increasing trend. Under GTS_ Traffic scene, while $\mathrm{SO}=\mathrm{BO}$ increases near median, the average energy consumption of network tends for convergence; under Traffic scene, the increasing trend shows linear slow-down.

\section{CONCLUSION}

The author makes use of OPNET simulation software to establish different working scenes via simulation, and carefully researches MAC layer in IEEE 802.15.4. The data comparison result shows that GTS mechanism has the lowest energy consumption and it can delay the network lifetime in actual work. In the simulation, in order to conveniently observe energy consumption, the small startype network architecture is adopted. The above analysis result can provide valuable reference for research on largescale WSN. However, it shall be noted that the service environment of WSN in reality is more complicated, thus the technical personnel shall design MAC protocol 
according to different site demands in the actual design process so as to reduce the energy consumption.

\section{REFERENCES}

[1] Ma Zuchang, Sun Yining: Research on Router Protocol of Largescale Wireless Sensor Network [J], Computer Engineering and Applications, 2014, 11: 165-168.
[2] Hamrita TK, Kaluskar NP and Wolfe KL. Advances in smart sensor technology[C]. Conference of Industry Applications, 2013,3: 2059-2062.

[3] Yuan Linfeng: Research on Energy Saving Strategies for Network Layer in Wireless Sensor Network [D], [Doctoral Dissertation], Huazhong University of Science and Technology, 2013.

[4] Chen Min: OPNET Network Simulation [M], Beijing: Tsinghua University Press, 2010. 